\title{
A Flexible Approach to (S)-5-Alkyl-tetramic acid Derivatives: Application to the Asymmetric Synthesis of (+)-Preussin and Protected (3S, 4S)-AHPPA
}

\author{
Pei-Qiang Huang, ${ }^{*}$ Tian-Jun Wu and Yuan-Ping Ruan \\ Department of Chemistry and The Key Laboratory for Chemical Biology of Fujian \\ Province, Xiamen University, Xiamen, Fujian 361005, P. R. China \\ E-mail: (pqhuang@xmu.edu.cn)
}

\section{General Information}

Optical rotations were recorded on a Perkin-Elmer 341 automatic polarimeter. ${ }^{1} \mathrm{H}-\mathrm{NMR}$ and ${ }^{13} \mathrm{C}$-NMR spectra were recorded on Varian unity +500 spectrometer. Unless otherwise noted, ${ }^{1} \mathrm{H}-\mathrm{NMR}$ spectra were registered in $\mathrm{CDCl}_{3}$, and chemical shifts are expressed in parts per million $(\delta)$ relative to internal $\mathrm{Me}_{4} \mathrm{Si}$. IR spectra were recorded on a Nicolet Avatar 360 FT-IR spectrophotometer. Mass spectra were recorded by Bruker Dalton Esquire 3000 plus and Finnigan Mat-LCQ (ESI direct injection). HRFABMS spectra were recorded on a Bruker APEX-FTMS apparatus. Elemental analyses were performed using a Vario RL analyzer. Melting points were determined on a Yanaco MP-500 melting point apparatus and are uncorrected.

Tetrahydrofuran was distilled prior to use from sodium benzophenone ketyl. Methylene chloride was distilled from phosphorus pentoxide. Silica gel (zhifu, 300-400 mesh) from Yantai silica gel factory (China) was used for column chromatography, eluting (unless otherwise stated) with ethyl acetate/petroleum ether (PE) $\left(60-90^{\circ} \mathrm{C}\right)$ mixture.

\section{4-Methoxy-1-(4-methoxybenzyl)-2,5-dihydro-1H-2-azolone (11) $\mathrm{MeO}$}

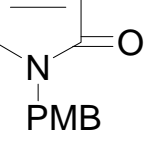

To a refluxing solution of 4-methoxybenzylamine (4.68 g, $34.2 \mathrm{mmol}$ ) in MeCN (17.5 $\mathrm{mL}$ ) were added dropwise methyl (E)-4-chloro-3-methoxy-2-butenoate $\mathbf{1 0}$ (4.5 g, 27.4 $\mathrm{mmol})$ in $\mathrm{MeCN}(17.5 \mathrm{~mL})$ and a solution of $\mathrm{Et}_{3} \mathrm{~N}(3.06 \mathrm{~g}, 30.25 \mathrm{mmol})$ in $\mathrm{MeCN}$ $(3.0 \mathrm{~mL})$ in parallel. After completed the additions, the reflux was maintained for $4 \mathrm{~h}$. The mixture was then chilled with an ice-bath, and the precipitate was filtered. The residue was washed with $\mathrm{MeCN}(30 \mathrm{~mL})$, and the combined filtrates were concentrated under reduced pressure. The residue was dissolved in $\mathrm{H}_{2} \mathrm{O}(15 \mathrm{~mL})$, acidified with conc. $\mathrm{HCl}$ until $\mathrm{pH} 3$. The resulting mixture was extracted with $\mathrm{CH}_{2} \mathrm{Cl}_{2}$. The combined extracts were concentrated under reduced pressure to give a residue, which was subjected to column chromatography on silica gel (EtOAc / $\mathrm{PE}=3 / 1$ ) to provided $11(4.524 \mathrm{~g}, 75.4 \%)$ as a white solid. Mp 63-65 ${ }^{\circ} \mathrm{C}$; IR (KBr) v $v_{\max }$ : 2936, 1682, 1513, 1455, 1353, $1264 \mathrm{~cm}^{-1} ;{ }^{1} \mathrm{H}-\mathrm{NMR}\left(500 \mathrm{MHz}, \mathrm{CDCl}_{3}\right) \delta: 3.68(\mathrm{~s}, 2 \mathrm{H}), 3.77$ $(\mathrm{s}, 3 \mathrm{H}), 3.80(\mathrm{~s}, 3 \mathrm{H}), 4.50(\mathrm{~s}, 2 \mathrm{H}), 5.07(\mathrm{~s}, 1 \mathrm{H}), 6.85(\mathrm{~d}, J=8.6 \mathrm{~Hz}, 2 \mathrm{H}), 7.17(\mathrm{~d}, J=$ $8.6 \mathrm{~Hz}, 2 \mathrm{H}) ;{ }^{13} \mathrm{C}-\mathrm{NMR}\left(125 \mathrm{MHz}, \mathrm{CDCl}_{3}\right) \delta: 173.35,171.99,158.93,129.42,129.23$, 114.00, 94.19, 58.04, 55.22, 49.72, 44.70; $\mathrm{MS}(\mathrm{m} / \mathrm{z}): 256.0\left(\mathrm{M}+\mathrm{Na}^{+}, 100\right), 234.0$ 
$\left(\mathrm{M}+\mathrm{H}^{+}, 23\right)$; Anal. Calcd for $\mathrm{C}_{13} \mathrm{H}_{15} \mathrm{NO}_{3}$ : C, 66.94; $\mathrm{H}, 6.48 ; \mathrm{N}, 6.00$. Found: $\mathrm{C}, 67.15$; $\mathrm{H}, 6.62 ; \mathrm{N}, 5.90$.

1-(4-Methoxybenzyl)-pyrrolidine-2,4-dione (12)

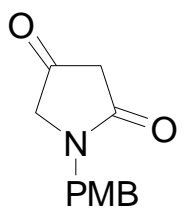

To $500 \mathrm{mg}$ ( $2.09 \mathrm{mmol})$ of finely pulverized 11 was added a $37 \% \mathrm{HCl}$ solution $(5 \mathrm{~mL})$. The resulting solution was stirred at $\mathrm{rt}$ for $5 \mathrm{~h}$, then extracted with $\mathrm{CH}_{2} \mathrm{Cl}_{2}$. The combined $\mathrm{CH}_{2} \mathrm{Cl}_{2}$ layers were dried over anhydrous $\mathrm{Na}_{2} \mathrm{SO}_{4}$, filtered and concentrated under reduced pressure. The crude was purified by chromatography on silica gel $(\mathrm{EtOAc} / \mathrm{PE}=1 / 1)$ to give $\mathbf{1 2}(432 \mathrm{mg}, 75 \%)$ as a pale yellow oil. IR $(\mathrm{KBr}) v_{\max }: 2921$, 1774, 1692, 1514, 1248, 1177, $1030 \mathrm{~cm}^{-1}$; ${ }^{1} \mathrm{H}-\mathrm{NMR}\left(500 \mathrm{MHz}, \mathrm{CDCl}_{3}\right.$ ) $\delta: 3.09$ (s, $2 \mathrm{H}), 3.72(\mathrm{~s}, 2 \mathrm{H}), 3.80(\mathrm{~s}, 3 \mathrm{H}), 4.57(\mathrm{~s}, 2 \mathrm{H}), 6.85(\mathrm{~d}, J=8.6 \mathrm{~Hz}, 2 \mathrm{H}), 7.20(\mathrm{~d}, J=$ $8.6 \mathrm{~Hz}, 2 \mathrm{H}) ;{ }^{13} \mathrm{C}-\mathrm{NMR}\left(125 \mathrm{MHz}, \mathrm{CDCl}_{3}\right) \delta: 203.3,168.6,159.5,129.8,127.0,114.3$, 56.6, 55.3, 45.4, 41.7; MS (m/z): $242\left(\mathrm{M}+\mathrm{Na}^{+}, 44\right), 220\left(\mathrm{M}+\mathrm{H}^{+}, 100\right)$; Anal. Calcd for $\mathrm{C}_{12} \mathrm{H}_{13} \mathrm{NO}_{3}$ : C, 65.74; H, 5.98; N, 6.39. Found: C, 65.92; H, 6.09; N, 6.55.

\section{4-[(S)-2-(1-Methoxy-1-ethylpropyl)pyrrolidin-1-yl]-1-(4-methoxybenzyl)-2,5-dihy} dro-1H-2-azolone (9)

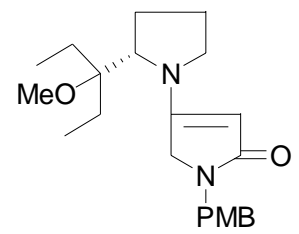

A solution of 12 (470 mg, $2.29 \mathrm{mmol}),(S)$-2-(1-methoxy-1-ethylpropyl)-pyrrolidine 13 (392 mg, $2.29 \mathrm{mmol}$ ), and a catalytic amount of PPTS in benzene (5 mL) was heated to reflux for $24 \mathrm{~h}$ with continuing elimination of water using a Dean-Stark apparatus. After removal of the solvent under reduced pressure, the resulting residue was purified by column chromatography on silica gel (EtOAc : $\mathrm{PE}=3: 1$ ) to give 9 $(427 \mathrm{mg}, 50.1 \%)$ as a colorless oil. $[\alpha]_{\mathrm{D}}{ }^{20}-27.8\left(c 1.2, \mathrm{CHCl}_{3}\right)$; IR (KBr) $v_{\max }: 2971$, 2936, 1666, 1596, 1512, 1457, 1393, 1246, 1174, 1081, $1030 \mathrm{~cm}^{-1} ;{ }^{1} \mathrm{H}-\mathrm{NMR}(500$ $\left.\mathrm{MHz}, \mathrm{CDCl}_{3}\right) \delta: 0.9\left(\mathrm{~m}, 6 \mathrm{H}, \mathrm{CH}_{3}\right), 1.40-2.02\left(\mathrm{~m}, 8 \mathrm{H}, \mathrm{CH}_{2}\right), 3.09\left(\mathrm{~s}, 3 \mathrm{H}, \mathrm{OCH}_{3}\right)$, $3.11-3.16(\mathrm{~m}, 1 \mathrm{H}), 3.22-3.28(\mathrm{~m}, 1 \mathrm{H}), 3.62(\mathrm{~m}, 1 \mathrm{H}), 3.68(\mathrm{~d}, J=16.9 \mathrm{~Hz}, 1 \mathrm{H}), 3.80$ $(\mathrm{s}, 3 \mathrm{H}), 4.00(\mathrm{~d}, J=16.9 \mathrm{~Hz}, 1 \mathrm{H}), 4.50(\mathrm{~s}, 2 \mathrm{H}), 4.75(\mathrm{~s}, 1 \mathrm{H}), 6.85(\mathrm{~d}, J=8.6 \mathrm{~Hz}$, $2 \mathrm{H}), 7.18(\mathrm{~d}, J=8.6 \mathrm{~Hz}, 2 \mathrm{H}) ;{ }^{13} \mathrm{C}-\mathrm{NMR}\left(125 \mathrm{MHz}, \mathrm{CDCl}_{3}\right) \delta: 174.2,162.7,158.7$, 130.4, 129.1, 113.9, 90.4, 81.5, 65.8, 55.2, 50.7, 49.3, 44.9, 27.0, 26.2, 23.9, 8.1, 7.4; HRMS (ESI) calcd for $\left[\mathrm{C}_{22} \mathrm{H}_{32} \mathrm{~N}_{2} \mathrm{O}_{3}+\mathrm{H}\right]^{+}: 373.2486$; found: 373.2480 .

\section{Representative procedure for the alkylation of $(S)-9$.}


To a solution of $(S)-9(120 \mathrm{mg}, 0.323 \mathrm{mmol})$ in THF [5.5 mL, containing $0.28 \mathrm{~mL}$ $(1.62 \mathrm{mmol})$ of HMPA as a co-solvent] was added dropwise $t$-BuLi $\quad(0.26 \mathrm{~mL}, 0.39$ mmol, $1.5 \mathrm{M}$ in pentane) at $-78^{\circ} \mathrm{C}$. After being stirred for $1 \mathrm{~h}$, methyl iodide $(0.2 \mathrm{~mL}$, $3.23 \mathrm{mmol}$ ) was added and the stirring continued for an additional $7 \mathrm{~h}$. The reaction was quenched by saturated ammonium chloride $(2 \mathrm{~mL})$. The resulting mixture was extracted with ether, and the organic layers were washed with brine, dried over $\mathrm{MgSO}_{4}$ and concentrated under reduced pressure. The crude was purified by column chromatography on silica gel to give 14b (109 $\mathrm{mg}, 87.4 \%)$ as a single regio and diastereomer.

(5RS)-4-[(S)-2-(1-Methoxy-1-ethylpropyl)pyrrolidin-1-yl]-1-(4-methoxybenzyl)-5 -deutero-2,5-dihydro-1H-2-azolone (14a)

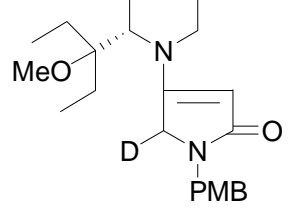

14a: Colorless oil. Yield: $78 \%$. $[\alpha]_{\mathrm{D}}{ }^{20}-26.1$ (c 2.0, $\left.\mathrm{CHCl}_{3}\right)$; IR (KBr) v $v_{\max }$ : 2928, 1665, 1590, 1512, 1392, 1245, $1033 \mathrm{~cm}^{-1}$; ${ }^{1} \mathrm{H}-\mathrm{NMR}\left(500 \mathrm{MHz}, \mathrm{CDCl}_{3}\right) \delta: 0.83(\mathrm{~m}, 6 \mathrm{H})$, 1.40-2.10 (m, $8 \mathrm{H}), 3.10(\mathrm{~s}, 3 \mathrm{H}), 3.12-3.17(\mathrm{~m}, 1 \mathrm{H}), 3.22-3.29(\mathrm{~m}, 1 \mathrm{H}), 3.60-3.64(\mathrm{~m}$, $1 \mathrm{H}), 3.68(\mathrm{~s}, 1 \mathrm{H}), 3.79(\mathrm{~s}, 3 \mathrm{H}), 4.51(\mathrm{~s}, 2 \mathrm{H}), 4.80(\mathrm{~s}, 1 \mathrm{H}), 6.83(\mathrm{~d}, J=8.6 \mathrm{~Hz}, 2 \mathrm{H})$, $7.18(\mathrm{~d}, J=8.6 \mathrm{~Hz}, 2 \mathrm{H}) ;{ }^{13} \mathrm{C}-\mathrm{NMR}\left(125 \mathrm{MHz}, \mathrm{CDCl}_{3}\right) \delta: 174.3,162.7,158.7,130.5$, 129.2, 113.9, 90.5, 81.5, 65.8, 55.3, 50.6, 49.4, 44.9, 27.0, 26.2, 24.0, 8.2, 7.5; MS $(\mathrm{m} / \mathrm{z}): 396\left(\mathrm{M}+\mathrm{Na}^{+}, 20\right), 374\left(\mathrm{M}+\mathrm{H}^{+}, 100\right)$; HRMS calcd for $\left[\mathrm{C}_{22} \mathrm{H}_{31} \mathrm{DN}_{2} \mathrm{O}_{3}+\mathrm{H}\right]^{+}$: 374.2548; found: 374.2539 .

(5S)-4-[(S)-2-(1-Methoxy-1-ethylpropyl)pyrrolidin-1-yl]-1-(4-methoxybenzyl)-5methyl-2,5-dihydro-1H-2-azolone (14b)

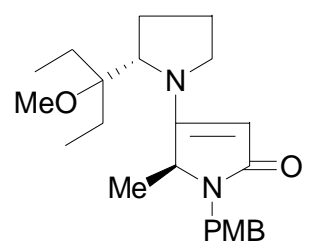

14b: Colorless oil. Yield: $87.4 \%$. $[\alpha]_{\mathrm{D}}{ }^{20}+30.2\left(c\right.$ 1.1, $\left.\mathrm{CHCl}_{3}\right)$; IR $(\mathrm{KBr}) v_{\max }$ : 2932, 1666, 1592, 1511, 1388, 1245, $1032 \mathrm{~cm}^{-1} ;{ }^{1} \mathrm{H}-\mathrm{NMR}\left(500 \mathrm{MHz}, \mathrm{CDCl}_{3}\right) \delta: 0.85(\mathrm{~m}$, $6 \mathrm{H}), 1.25(\mathrm{~d}, J=6.3 \mathrm{~Hz}, 3 \mathrm{H}), 1.36-2.08(\mathrm{~m}, 8 \mathrm{H}), 3.10-3.17(\mathrm{~m}, 1 \mathrm{H}), 3.2(\mathrm{~s}, 3 \mathrm{H})$, 3.26-3.33 (m, 1H), $3.68(\mathrm{~m}, 1 \mathrm{H}), 3.78(\mathrm{~s}, 3 \mathrm{H}), 3.80-3.87(\mathrm{~m}, 1 \mathrm{H}), 3.92(\mathrm{~d}, J=15.3 \mathrm{~Hz}$, $1 \mathrm{H}), 4.88(\mathrm{~s}, 1 \mathrm{H}), 5.08(\mathrm{~d}, J=15.3 \mathrm{~Hz}, 1 \mathrm{H}), 6.85(\mathrm{~d}, J=8.5 \mathrm{~Hz}, 2 \mathrm{H}), 7.18(\mathrm{~d}, J=8.5$ $\mathrm{Hz}, 2 \mathrm{H}) ;{ }^{13} \mathrm{C}-\mathrm{NMR}\left(125 \mathrm{MHz}, \mathrm{CDCl}_{3}\right) \delta: 172.6,168.4,158.7,130.3,129.1,113.9$, 91.4, 81.5, 66.1, 55.7, 55.2, 51.2, 50.2, 42.2, 26.6, 25.8, 24.7, 17.0, 8.4, 7.8; HRMS (ESI) calcd for $\left[\mathrm{C}_{23} \mathrm{H}_{34} \mathrm{~N}_{2} \mathrm{O}_{3}+\mathrm{H}\right]^{+}: 387.2642$; found: 387.2635 . 


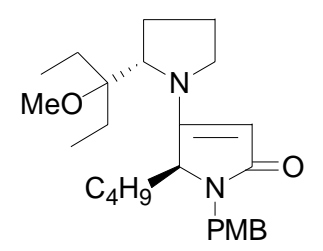

14c: Colorless oil. Yield: $71 \%$. $[\alpha]_{\mathrm{D}}{ }^{20}+29.7\left(c 1.1, \mathrm{CHCl}_{3}\right)$; IR (KBr) $v_{\max }$ : 2957, 2922, 1663, 1591, 1509, 1407, 1242, $1026 \mathrm{~cm}^{-1} ;{ }^{1} \mathrm{H}-\mathrm{NMR}\left(500 \mathrm{MHz}, \mathrm{CDCl}_{3}\right) \delta: 0.85(\mathrm{~m}$, $9 \mathrm{H}), 1.08-2.06(\mathrm{~m}, 14 \mathrm{H}), 3.13-3.19(\mathrm{~m}, 1 \mathrm{H}), 3.21(\mathrm{~s}, 3 \mathrm{H}), 3.28-3.35(\mathrm{~m}, 1 \mathrm{H})$, 3.64-3.73 (m, 1H), 3.79 (s, 3H), $3.85(\mathrm{~d}, J=15.2 \mathrm{~Hz}, 1 \mathrm{H}), 3.93(\mathrm{~m}, 1 \mathrm{H}), 4.95(\mathrm{~s}, 1 \mathrm{H})$, $5.11(\mathrm{~d}, J=15.2 \mathrm{~Hz}, 1 \mathrm{H}), 6.82(\mathrm{~d}, J=8.5 \mathrm{~Hz}, 2 \mathrm{H}), 7.18(\mathrm{~d}, J=8.5 \mathrm{~Hz}, 2 \mathrm{H}) ;{ }^{13} \mathrm{C}-\mathrm{NMR}$ $\left(125 \mathrm{MHz}, \mathrm{CDCl}_{3}\right) \delta: 173.4,166.6,158.8,130.3,129.2,113.9,93.1,81.5,66.4,59.4$, 55.3, 51.7, 50.1, 42.53, 28.4, 26.6, 25.7, 24.9, 24.7, 24.0, 22.6, 14.0, 8.4, 7.8; HRMS (ESI) calcd for $\left[\mathrm{C}_{26} \mathrm{H}_{40} \mathrm{~N}_{2} \mathrm{O}_{3}+\mathrm{H}\right]^{+}: 429.2642$; found: 429.2635; Anal. Calcd for $\mathrm{C}_{26} \mathrm{H}_{40} \mathrm{~N}_{2} \mathrm{O}_{3}$ : C, 72.86; H, 9.41; N, 6.54. Found: C, 73.06; H, 9.42; N, 6.60.

(5S)-4-[(S)-2-(1-Methoxy-1-ethylpropyl)pyrrolidin-1-yl]-1-(4-methoxybenzyl)-5methyl-2,5-dihydro-1H-2-azolone (14d)

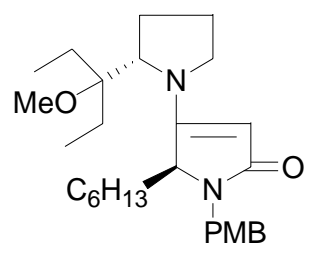

14d: Colorless oil. Yield: $69 \%$. $[\alpha]_{\mathrm{D}}^{20}+38.8\left(c 1.9, \mathrm{CHCl}_{3}\right)$; IR $(\mathrm{KBr}) v_{\max }$ : 2931, 1667, 1593, 1512, 1382, 1246, 1174, $1090 \mathrm{~cm}^{-1} ;{ }^{1} \mathrm{H}-\mathrm{NMR}\left(500 \mathrm{MHz}, \mathrm{CDCl}_{3}\right) \delta: 0.85$ $(\mathrm{m}, 9 \mathrm{H}), 1.08-2.06(\mathrm{~m}, 18 \mathrm{H}), 3.12-3.18(\mathrm{~m}, 1 \mathrm{H}), 3.20(\mathrm{~s}, 3 \mathrm{H}), 3.26-3.34(\mathrm{~m}, 1 \mathrm{H})$, 3.63-3.70 (m, $1 \mathrm{H}), 3.8(\mathrm{~s}, 3 \mathrm{H}), 3.84(\mathrm{~d}, J=15.2 \mathrm{~Hz}, 1 \mathrm{H}), 3.88-3.94(\mathrm{~m}, 1 \mathrm{H}), 4.94(\mathrm{~s}$, $1 \mathrm{H}), 5.09(\mathrm{~d}, J=15.2 \mathrm{~Hz}, 1 \mathrm{H}), 6.82(\mathrm{~d}, J=8.5 \mathrm{~Hz}, 2 \mathrm{H}), 7.18(\mathrm{~d}, J=8.5 \mathrm{~Hz}, 2 \mathrm{H})$; ${ }^{13} \mathrm{C}-\mathrm{NMR}\left(125 \mathrm{MHz}, \mathrm{CDCl}_{3}\right)$ \&: 173.3, 166.6, 158.7, 130.3, 129.3, 113.9, 93.2, 81.5, 66.4, 59.4, 55.3, 51.7, 50.2, 42.5, 31.8, 29.2, 28.7, 26.7, 25.7, 25.0, 24.7, 22.5, 21.8, 14.1, 8.5, 7.8; Anal. Calcd for $\mathrm{C}_{28} \mathrm{H}_{44} \mathrm{~N}_{2} \mathrm{O}_{3}$ : C, 73.64; H, 9.71; N, 6.13. Found: C, $73.28 ; \mathrm{H}, 9.74 ; \mathrm{N}, 6.38$.

(5S)-4-[(S)-2-(1-Methoxy-1-ethylpropyl)pyrrolidin-1-yl]-1-(4-methoxybenzyl)-5allyl-2,5-dihydro-1H-2-azolone (14e)

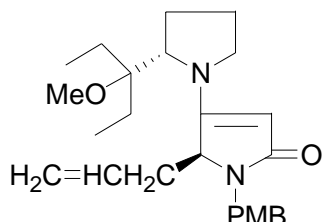

14e: Pale yellow oil. Yield: $77 \%$. $[\alpha]_{\mathrm{D}}{ }^{20}+39.9$ (c 1.2, $\left.\mathrm{CHCl}_{3}\right)$; IR $(\mathrm{KBr}) v_{\max }$ : 2929, $1667,1592,1512,1379,1246,1175 \mathrm{~cm}^{-1} ;{ }^{1} \mathrm{H}-\mathrm{NMR}\left(500 \mathrm{MHz}, \mathrm{CDCl}_{3}\right) \delta: 0.86(\mathrm{~m}$, 
$6 \mathrm{H}), 1.20-2.10(\mathrm{~m}, 8 \mathrm{H}), 2.38-2.48(\mathrm{~m}, 1 \mathrm{H}), 2.58-2.70(\mathrm{~m}, 1 \mathrm{H}), 3.16(\mathrm{~m}, 1 \mathrm{H}), 3.20(\mathrm{~s}$, $3 \mathrm{H}), 3.64-3.73(\mathrm{~m}, 1 \mathrm{H}), 3.80(\mathrm{~s}, 3 \mathrm{H}), 3.88(\mathrm{~d}, J=15.3 \mathrm{~Hz}, \mathrm{H}), 3.94(\mathrm{~m}, 1 \mathrm{H}), 4.93$ (s, $1 \mathrm{H}), 5.10(\mathrm{~m}, 2 \mathrm{H}), 5.17(\mathrm{~d}, J=15.3 \mathrm{~Hz}, 1 \mathrm{H}), 5.60(\mathrm{~m}, 1 \mathrm{H}), 6.82(\mathrm{~d}, J=8.5 \mathrm{~Hz}, 2 \mathrm{H})$, $7.18(\mathrm{~d}, J=8.5 \mathrm{~Hz}, 2 \mathrm{H}) ;{ }^{13} \mathrm{C}-\mathrm{NMR}\left(125 \mathrm{MHz}, \mathrm{CDCl}_{3}\right) \delta: 173.1,166.3,158.7,157.3$, 138.9, 131.4, 129.2, 118.7, 113.9, 110.9, 93.0, 92.8, 86.0, 81.6, 66.5, 58.8, 58.8, 56.4, $55.2,51.6,50.5,42.5,41.9,33.5,26.6,25.7,24.9,24.5,8.5,7.8 ; \mathrm{MS}(\mathrm{m} / \mathrm{z}): 435$ $\left(\mathrm{M}+\mathrm{Na}^{+}, 16\right), 413\left(\mathrm{M}+\mathrm{H}^{+}, 100\right)$; HRMS calcd for $\left[\mathrm{C}_{25} \mathrm{H}_{36} \mathrm{~N}_{2} \mathrm{O}_{3}+\mathrm{H}\right]^{+}: 413.2799$; found: 413.2797.

(5S)-4-[(S)-2-(1-Methoxy-1-ethylpropyl)pyrrolidin-1-yl]-1-(4-methoxybenzyl)-5ethyoxylcarboxylethyl-2,5-dihydro-1H-2-azolone (14f)

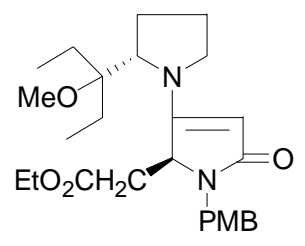

14f: Pale yellow oil. Yield: $84.2 \%$. $[\alpha]_{\mathrm{D}}{ }^{20}+11.8\left(c 0.7, \mathrm{CHCl}_{3}\right)$; IR $(\mathrm{KBr}) v_{\max }$ : 2925, 1733, 1671, 1596, 1377, 1246, 1174, $1033 \mathrm{~cm}^{-1} ;{ }^{1} \mathrm{H}-\mathrm{NMR}\left(500 \mathrm{MHz}, \mathrm{CDCl}_{3}\right.$, two rotamers, $\mathrm{M}: \mathrm{m}=11: 1) \delta: 0.85(\mathrm{~m}, 6 \mathrm{H}), 1.24(\mathrm{t}, J=7.2 \mathrm{~Hz}, 3 \mathrm{H}), 1.26-2.08(\mathrm{~m}, 8 \mathrm{H})$, 2.42-2.52 (dd, $J=15.5,7.2 \mathrm{~Hz}, 1 \mathrm{H}, \mathrm{M}), 2.54-2.60(\mathrm{dd}, J=15.5,8.8 \mathrm{~Hz}, 1 \mathrm{H}, \mathrm{m})$, 2.65-2.71 (dd, $J=15.5,5.2 \mathrm{~Hz}, 1 \mathrm{H}, \mathrm{m}), 2.76-2.84(\mathrm{~d}, \mathrm{brd}, J=15.5 \mathrm{~Hz}, 1 \mathrm{H}, \mathrm{M})$, 3.16-3.22 (m, 1H), $3.23(\mathrm{~s}, 3 \mathrm{H}), 3.28-3.36(\mathrm{~m}, 1 \mathrm{H}), 3.66-3.76(\mathrm{~m}, 1 \mathrm{H}), 3.80(\mathrm{~s}, 3 \mathrm{H})$, 4.12 (q, $J=7.2 \mathrm{~Hz}, 2 \mathrm{H}), 4.13(\mathrm{~m}, 1 \mathrm{H}), 4.36-4.42(\mathrm{~m}, 1 \mathrm{H}), 4.92$ (d, $J=15.4 \mathrm{~Hz}, 1 \mathrm{H}, \mathrm{M}$ and m overlapped), $4.98(\mathrm{~s}, 1 \mathrm{H}), 6.82(\mathrm{~d}, J=8.5 \mathrm{~Hz}, 2 \mathrm{H}), 7.20(\mathrm{~d}, J=8.5 \mathrm{~Hz}, 2 \mathrm{H})$; ${ }^{13} \mathrm{C}-\mathrm{NMR}\left(125 \mathrm{MHz}, \mathrm{CDCl}_{3}\right) \delta$ (two rotamers): 173.2, 170.7, 166.5, 158.7, 130.4, $129.1,114.0,113.8,93.9,92.9,81.6,66.3,61.2,61.0,60.4,58.3,57.3,56.5,55.2$, 53.4, 51.5, 50.4, 43.4, 43.0, 38.1, 35.6, 26.6, 25.9, 24.9, 24.7, 14.2, 14.1, 8.6, 7.9; HRMS (ESI) calcd for $\left[\mathrm{C}_{26} \mathrm{H}_{38} \mathrm{~N}_{2} \mathrm{O}_{5}+\mathrm{H}\right]^{+}: 459.2853$; found: 459.2853 .

(5S)-4-[(S)-2-(1-Methoxy-1-ethylpropyl)pyrrolidin-1-yl]-1-(4-methoxybenzyl)5-benzyl-2,5-dihydro-1 $H$-2-azolone (14g)

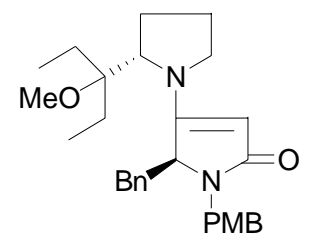

14g: Colorless oil. Yield: $85.4 \%$. $[\alpha]_{\mathrm{D}}^{20}+66.8\left(c\right.$ 1.1, $\left.\mathrm{CHCl}_{3}\right)$; IR $(\mathrm{KBr}) v_{\max }$ : 2926, 2856, 1698, 1512, 1248, $1113 \mathrm{~cm}^{-1} ;{ }^{1} \mathrm{H}-\mathrm{NMR}\left(500 \mathrm{MHz}, \mathrm{CDCl}_{3}\right.$, two rotamers, M: $\mathrm{m}=2.1: 1) \delta: 0.85(\mathrm{~m}, 6 \mathrm{H}), 1.20-2.10(\mathrm{~m}, 8 \mathrm{H}), 2.66-2.71(\mathrm{~m}, 2 \mathrm{H}, \mathrm{m}), 2.75(\mathrm{dd}, J=$ 15.1, 7.5 Hz, 2H, M), 3.15 (s, 3H), 3.20-3.34 (m, 2H), $3.50(\mathrm{~d}, J=15.1 \mathrm{~Hz}, 1 \mathrm{H}), 3.63$ (m, 1H), $3.75(\mathrm{~s}, 3 \mathrm{H}), 4.05(\mathrm{~m}, 1 \mathrm{H}), 4.90(\mathrm{~s}, 1 \mathrm{H}), 5.12(\mathrm{~d}, J=15.1 \mathrm{~Hz}, 1 \mathrm{H}), 6.72(\mathrm{~d}$, $J=7.7 \mathrm{~Hz}, 2 \mathrm{H}), 6.80(\mathrm{~d}, J=7.7 \mathrm{~Hz}, 2 \mathrm{H}), 7.15-7.35$ (m, 5H); ${ }^{13} \mathrm{C}-\mathrm{NMR}(125 \mathrm{MHz}$, 
$\left.\mathrm{CDCl}_{3}\right) \delta$ (two rotamers): $173.5,167.1,158.6,136.9,129.9,129.2,128.5,126.9$, 113.7, 93.1, 81.6, 66.4, 60.3, 55.1, 51.6, 50.6, 43.2, 38.0, 26.5, 25.6, 24.8, 24.2, 8.6, 7.8; $\mathrm{MS}(\mathrm{m} / \mathrm{z}): 485\left(\mathrm{M}+\mathrm{Na}^{+}, 22\right), 463\left(\mathrm{M}+\mathrm{H}^{+}, \quad 100\right)$; HRMS calcd for $\left[\mathrm{C}_{29} \mathrm{H}_{38} \mathrm{~N}_{2} \mathrm{O}_{3}+\mathrm{H}\right]^{+}:$463.2955; found: 463.2960 .

(5S)-4-[(S)-2-(1-Methoxy-1-ethylpropyl)pyrrolidin-1-yl]-1-(4-methoxybenzyl)-5trimethylsilyl-2,5-dihydro-1H-2-azolone (14h)

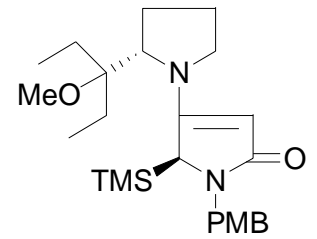

14h: Pale yellow oil. Yield: $45 \%$. $[\alpha]_{\mathrm{D}}^{20}-13.1\left(c 0.8, \mathrm{CHCl}_{3}\right)$; IR $(\mathrm{KBr}) v_{\max }$ : 2920, 1652, 1597, 1463, 1398, 1239, 1090, $836(\mathrm{~s}) \mathrm{cm}^{-1}$; ${ }^{1} \mathrm{H}-\mathrm{NMR}\left(500 \mathrm{MHz}, \mathrm{CDCl}_{3}\right) \delta$ : $0.22(\mathrm{~s}, 9 \mathrm{H}), 0.83(\mathrm{~m}, 6 \mathrm{H}), 1.40-2.10(\mathrm{~m}, 8 \mathrm{H}), 3.09(\mathrm{~s}, 3 \mathrm{H}), 3.10-3.18(\mathrm{~m}, 1 \mathrm{H})$, 3.22-3.29 (m, 1H), 3.60-3.66 (m, $1 \mathrm{H}), 3.70(\mathrm{~d}, J=17.0 \mathrm{~Hz}, 1 \mathrm{H}), 3.78(\mathrm{~s}, 3 \mathrm{H}), 4.50$ (dd, $J=15.9 \mathrm{~Hz}, 2 \mathrm{H}), 4.79$ (s, 1H), 6.77 (d, $J=8.6 \mathrm{~Hz}, 2 \mathrm{H}), 7.20$ (d, $J=8.6 \mathrm{~Hz}, 2 \mathrm{H})$. ${ }^{13} \mathrm{C}-\mathrm{NMR}\left(125 \mathrm{MHz}, \mathrm{CDCl}_{3}\right) \delta: 174.2,163.6,162.8,134.6,130.5,129.9,127.9,109.7$, 90.6, 81.5, 65.8, 55.1, 50.8, 50.7, 49.4, 45.2, 29.7, 27.0, 26.3, 26.2, 24.0, 8.2 , 7.5, -1.0; MS (m/z): $445\left(\mathrm{M}+\mathrm{H}^{+}, 100\right)$; HRMS calcd for $\left[\mathrm{C}_{25} \mathrm{H}_{40} \mathrm{SiN}_{2} \mathrm{O}_{3}+\mathrm{H}\right]^{+}: 445.2881$; found: 445.2879 .

(4S, 5S)-5-Benzyl-4-hydroxy-1-(4-methoxybenzyl)-2-pyrrolidinone (16)

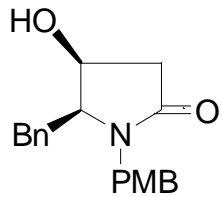

To a solution of $\mathbf{1 4 g}$ ( $727 \mathrm{mg}, 1.57 \mathrm{mmol})$ in THF (50 mL) was added a solution of 10 $\% \mathrm{HCl}(20 \mathrm{~mL})$. After stirred at $30^{\circ} \mathrm{C}$ for $26 \mathrm{~h}$, the mixture was extracted with ethyl acetate and the combined extracts were washed with brine, dried over anhydrous $\mathrm{MgSO}_{4}$, filtered, and concentrated under reduced pressure. The residue was dissolved in a mixed solvent system $\left(\mathrm{CH}_{2} \mathrm{Cl}_{2}, 11.5 \mathrm{~mL}\right.$; $\left.\mathrm{AcOH}, 1.2 \mathrm{~mL}\right)$, then cooled to $0{ }^{\circ} \mathrm{C}$ and stirred vigorously, into which was added portion-wise $\mathrm{NaBH}_{4}(141 \mathrm{mg}, 3.7 \mathrm{mmol})$. After stirred for $3.5 \mathrm{~h}$, a cold saturated solution of $\mathrm{NaHCO}_{3}(3 \mathrm{~mL})$ was added. The resulting mixture was extracted with $\mathrm{CH}_{2} \mathrm{Cl}_{2}$. The combined organic layers were washed with brine, dried $\left(\mathrm{MgSO}_{4}\right)$, filtered and concentrated in vacuo. The crude was purified by column chromatography on silica gel (EtOAc / $\mathrm{PE}=1 / 1)$ to give $(4 S$, $5 S)-16(400 \mathrm{mg}, 82 \%)$ and $(4 R, 5 S)-16$ as an un-separable mixture in a ratio of 20:1 (deduced from 17). (4S, 5S)-16: White solid. Mp 144.5-145.5 ${ }^{\circ} \mathrm{C} ;[\alpha]_{\mathrm{D}}{ }^{20}-75.7$ (c 0.7, $\mathrm{CHCl}_{3}$ ); IR (KBr) v $v_{\max }: 2926,2856,1698,1512,1248,1113 \mathrm{~cm}^{-1} ;{ }^{1} \mathrm{H}-\mathrm{NMR}(500 \mathrm{MHz}$, $\left.\mathrm{CD}_{3} \mathrm{CN}\right) \delta: 2.28(\mathrm{dd}, J=16.5,3.7 \mathrm{~Hz}, 1 \mathrm{H}), 2.56(\mathrm{dd}, J=16.5,6.1 \mathrm{~Hz}, 1 \mathrm{H}), 2.97(\mathrm{dd}$, 
$J=13.4,5.5 \mathrm{~Hz}, 1 \mathrm{H}), 3.04(\mathrm{dd}, J=13.4,8.6 \mathrm{~Hz}, 1 \mathrm{H}), 3.33\left(\mathrm{~d}, J=5.5 \mathrm{~Hz}, 1 \mathrm{H}, \mathrm{D}_{2} \mathrm{O}\right.$ exchangeable), 3.70 (ddd, $J=9.2,6.1,3.7 \mathrm{~Hz}, 1 \mathrm{H}), 3.83(\mathrm{~s}, 3 \mathrm{H}), 3.97$ (d, $J=15.3 \mathrm{~Hz}$, 1H), $4.15(\mathrm{~m}, 1 \mathrm{H}), 4.85$ (d, $J=15.3 \mathrm{~Hz}, 1 \mathrm{H}), 6.94$ (d, $J=8.6 \mathrm{~Hz}, 2 \mathrm{H}), 7.16$ (d, $J=8.6$ $\mathrm{Hz}, 2 \mathrm{H}), 7.20-7.38$ (m, 5H); ${ }^{13} \mathrm{C}-\mathrm{NMR}\left(125 \mathrm{MHz}, \mathrm{CDCl}_{3}\right) \delta: 173.4,159.0,137.4$, $129.2,129.2,128.7,128.4,126.7,114.1,66.1,62.8,55.3,43.6,40.3,32.9 ; \mathrm{MS}(\mathrm{m} / \mathrm{z})$ : $334\left(\mathrm{M}+\mathrm{Na}^{+}, 100\right), 311\left(\mathrm{M}+\mathrm{H}^{+}, 19\right)$; Anal. Calcd for $\mathrm{C}_{19} \mathrm{H}_{21} \mathrm{NO}_{3}: \mathrm{C}, 73.31 ; \mathrm{H}, 6.75 ; \mathrm{N}$, 4.50. Found: C, 73.24; H, 6.80; N, 4.67.

(4S, 5S)-5-Benzyl-4-(tert-butyldimethylsilyoxy)-1-(4-methoxybenzyl)-2-pyrrolidinone (17)

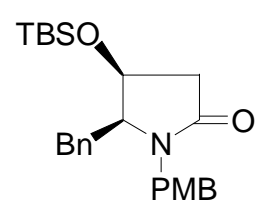

To a mixture of 16 (400 mg, $1.29 \mathrm{mmol})$, imidazole (175 mg, $2.57 \mathrm{mmol})$ and a catalytic amount of DMAP in anhydrous $\mathrm{CH}_{2} \mathrm{Cl}_{2}(8 \mathrm{~mL})$ was added a solution of tert-butyldimethylchlorosilane $(387 \mathrm{mg}, 2.57 \mathrm{mmol})$ in anhydrous $\mathrm{CH}_{2} \mathrm{Cl}_{2}(4 \mathrm{~mL})$. After being stirred at $\mathrm{rt}$ overnight, an aqueous solution of $\mathrm{NaHCO}_{3}(10 \mathrm{~mL})$ was added. The organic layer was separated, and washed with brine, dried over anhydrous $\mathrm{Na}_{2} \mathrm{SO}_{4}$ and concentrated. The crude was purified by column chromatography on silica gel (EtOAc / $\mathrm{PE}=1 / 5)$ to give $17(514 \mathrm{mg}, 90 \%)$ as a colorless oil. $[\alpha]_{\mathrm{D}}{ }^{20}-31.6$ (c 1.0, $\mathrm{CHCl}_{3}$ ); IR (KBr) v $v_{\max }: 2926,2856,1698,1512,1248,1113 \mathrm{~cm}^{-1}$; ${ }^{1} \mathrm{H}-\mathrm{NMR}$ $\left(500 \mathrm{MHz}, \mathrm{CDCl}_{3}\right) \delta:-0.03(\mathrm{~s}, 3 \mathrm{H}), 0.03(\mathrm{~s}, 3 \mathrm{H}), 0.90(\mathrm{~s}, 9 \mathrm{H}), 2.37$ (dd, $J=16.3,7.4$ $\mathrm{Hz}, 1 \mathrm{H}), 2.49(\mathrm{dd}, J=16.3,7.1 \mathrm{~Hz}, 1 \mathrm{H}), 2.81(\mathrm{dd}, J=14.3,7.1 \mathrm{~Hz}, 1 \mathrm{H}), 3.08(\mathrm{dd}, J=$ $14.3,5.8 \mathrm{~Hz}, 1 \mathrm{H}), 3.48(\mathrm{~d}, J=14.9 \mathrm{~Hz}, 1 \mathrm{H}), 3.70(\mathrm{ddd}, J=7.1,7.1,5.8 \mathrm{~Hz}, 1 \mathrm{H}), 3.78$ (s, 3H), $4.32(\mathrm{ddd}, J=7.4,7.1,7.1 \mathrm{~Hz}, 1 \mathrm{H}), 4.93(\mathrm{~d}, J=14.9 \mathrm{~Hz} 1 \mathrm{H}), 6.80(\mathrm{~d}, J=8.6$ $\mathrm{Hz}, 2 \mathrm{H}), 6.90(\mathrm{~d}, J=8.6 \mathrm{~Hz}, 2 \mathrm{H}), 7.15-7.35(\mathrm{~m}, 5 \mathrm{H}) ;{ }^{13} \mathrm{C}-\mathrm{NMR}\left(125 \mathrm{MHz}, \mathrm{CDCl}_{3}\right) \delta$ : $172.3,158.9,138.4,129.4,129.2,128.6,128.5,126.5,113.9,67.9,62.3,55.2,43.8$, 39.5, 33.8, 25.7, 18.0, -4.5, -5.1; MS (m/z): $426\left(\mathrm{M}+\mathrm{H}^{+}, 100\right)$; HRMS calcd for $\left[\mathrm{C}_{25} \mathrm{H}_{35} \mathrm{NO}_{3} \mathrm{Si}+\mathrm{H}\right]^{+}:$426.2459; found: 426.2457.

\section{(4S, 5S)-5-Benzyl-4-(tert-butyldimethylsilyloxy)-2-pyrrolidinone (18)}

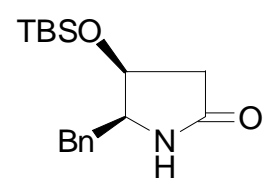

To a solution of 17 (389 mg, $0.915 \mathrm{mmol})$ in a mixed $\mathrm{CH}_{3} \mathrm{CN}(11.4 \mathrm{~mL})$ and $\mathrm{H}_{2} \mathrm{O}(3.8$ $\mathrm{mL})$ solvent system was added ceric ammonium nitrate $(2.0 \mathrm{~g}, 3.66 \mathrm{mmol})$ in one portion. After stirred at $\mathrm{rt}$ for $25 \mathrm{~min} ., \mathrm{H}_{2} \mathrm{O}(10 \mathrm{~mL})$ was added and the mixture was extracted with EtOAc $(30 \mathrm{~mL} \times 3)$. The combined organic layers were washed successively with saturated aqueous $\mathrm{NaHCO}_{3}(5 \mathrm{~mL} \times 3)$ and brine $(5 \mathrm{~mL})$. The organic phase was dried over anhydrous $\mathrm{Na}_{2} \mathrm{SO}_{4}$, filtrated and concentrated. The crude was purified by chromatography on silica gel $($ EtOAc / $\mathrm{PE}=1 / 1)$ to give $\mathbf{1 8}$ 
(202 mg, $74.6 \%$ ) as a colorless oil. $[\alpha]_{\mathrm{D}}^{20}-64\left(c 1.1, \mathrm{CHCl}_{3}\right)$; IR (KBr) $v_{\max }$ : 3389, 2927, 1702, 1255, 1149, $1082 \mathrm{~cm}^{-1} ;{ }^{1} \mathrm{H}-\mathrm{NMR}\left(500 \mathrm{MHz}, \mathrm{CDCl}_{3}\right) \delta: 0.10(\mathrm{~s}, 6 \mathrm{H}), 0.95$ (s, 9H), $2.34(\mathrm{dd}, J=16.7,4.5 \mathrm{~Hz}, 1 \mathrm{H}), 2.56(\mathrm{dd}, J=16.7,6.6 \mathrm{~Hz}, 1 \mathrm{H}), 2.74(\mathrm{dd}, J=$ $13.8,10.8 \mathrm{~Hz}, 1 \mathrm{H}$ ), 2.89-2.96 (dd, $J=13.8,3.7 \mathrm{~Hz}, 1 \mathrm{H}$ ), 3.85 (ddd, $J=10.8,5.4,3.7$ $\mathrm{H}, 1 \mathrm{H}), 4.56(\mathrm{ddd}, J=6.6,5.4,4.5 \mathrm{~Hz}, 1 \mathrm{H}), 5.40(\mathrm{~s}, 1 \mathrm{H}), 7.18-7.35(\mathrm{~m}, 5 \mathrm{H})$; ${ }^{13} \mathrm{C}-\mathrm{NMR}\left(125 \mathrm{MHz}, \mathrm{CDCl}_{3}\right) \delta: 174.8,138.2,129.0,128.9,126.7,69.3,60.7,40.3$, 36.2, 25.7, 18.1, -4.6, -5.0; MS (m/z): $306\left(\mathrm{M}+\mathrm{H}^{+}, 100\right)$; Anal. Calcd for $\mathrm{C}_{17} \mathrm{H}_{27} \mathrm{SiNO}_{2}$ : C, 66.84; H, 8.91; N, 4.59. Found: C, 67.11; H, 9.11; N, 4.76.

(4S, 5S)-5-Benzyl-4-(tert-butyldimethylsilyloxy)-1-(tert-butyloxycarbonyl)-2pyrrolidinone (19)

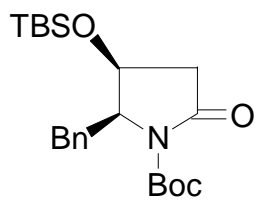

To a cooled $\left(0{ }^{\circ} \mathrm{C}\right)$ solution of $\mathbf{1 8}(202 \mathrm{mg}, 0.66 \mathrm{mmol})$ in $\mathrm{CH}_{2} \mathrm{Cl}_{2}(4 \mathrm{~mL})$ were added successively a catalytic amount of DMAP, $\mathrm{Et}_{3} \mathrm{~N}(0.18 \mathrm{~mL}, 1.32 \mathrm{mmol})$, and di(tert-butyl)dicarbonate $(289 \mathrm{mg}, 1.32 \mathrm{mmol})$. The mixture was allowed to stirr at $\mathrm{rt}$ overnight. After being diluted with $\mathrm{H}_{2} \mathrm{O}(3 \mathrm{~mL})$, the resulting mixture was extracted with $\mathrm{CH}_{2} \mathrm{Cl}_{2}$. The combined organic layers were washed with brine, dried, filtered and concentrated. The crude was purified by chromatography on silica gel (EtOAc / $\mathrm{PE}=1 / 10)$ to give $19(261 \mathrm{mg}, 97.3 \%)$ as a white solid. Mp $82.5-84.5{ }^{\circ} \mathrm{C} ;[\alpha]_{\mathrm{D}}{ }^{20}+34.3$ (c 1.0, $\mathrm{CHCl}_{3}$ ); IR (KBr) v $v_{\max }: 2956,2930,1791,1758,1715,1357,1295,1150 \mathrm{~cm}^{-1}$; ${ }^{1} \mathrm{H}-\mathrm{NMR}\left(500 \mathrm{MHz}, \mathrm{CDCl}_{3}\right) \delta: 0.02(\mathrm{~s}, 6 \mathrm{H}), 0.88(\mathrm{~s}, 9 \mathrm{H}), 1.45(\mathrm{~s}, 9 \mathrm{H}), 2.25(\mathrm{dd}, J=$ 16.8, 10.3 Hz, 1 H), $2.44(\mathrm{dd}, J=16.8,7.7 \mathrm{~Hz}, 1 \mathrm{H}), 2.94(\mathrm{dd}, J=14.1,5.1 \mathrm{~Hz}, 1 \mathrm{H})$, $3.18(\mathrm{dd}, J=14.1,5.7 \mathrm{~Hz}, 1 \mathrm{H}), 4.42-4.47$ (ddd, $J=7.5,5.7,5.1 \mathrm{~Hz}, 1 \mathrm{H}), 4.49-4.55$ $(\mathrm{ddd}, J=10.3,7.7,7.5 \mathrm{~Hz}, 1 \mathrm{H}), 7.15-7.35(\mathrm{~m}, 5 \mathrm{H}) ;{ }^{13} \mathrm{C}-\mathrm{NMR}\left(125 \mathrm{MHz}, \mathrm{CDCl}_{3}\right) \delta$ : 171.0, 149.6, 137.7, 130.1, 128.3, 126.5, 83.0, 66.9, 62.2, 40.4, 34.0, 27.9, 25.8, 18.1, -4.8, -5.0; MS (m/z): 348 (88), 391 (100), $404\left(\mathrm{M}+\mathrm{H}^{+}, 51\right), 426$ (19); Anal. Calcd for $\mathrm{C}_{22} \mathrm{H}_{35} \mathrm{NO}_{4} \mathrm{Si}: \mathrm{C}, 65.19 ; \mathrm{H}, 8.64 ; \mathrm{N}, 3.46$. Found: $\mathrm{C}, 65.35 ; \mathrm{H}, 8.74 ; \mathrm{N}, 3.70$.

(2S, 3S, 5R)-2-Benzyl-3-(tert-butyldimethylsilyloxy)-1-(tert-butyloxycarbonyl)-5(n-nonyl)-pyrrolidine (20)

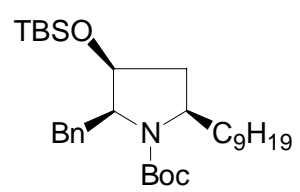

The transformation of $\mathbf{1 9}$ to $\mathbf{2 0}$ was achieved by reported procedure, ${ }^{2,3}$ which afforded $20(48 \mathrm{mg}, 75 \%)$ as a colorless oil. $[\alpha]_{\mathrm{D}}{ }^{20}-48.6\left(c 1.1, \mathrm{CHCl}_{3}\right)$. IR (KBr) $v_{\max }$ : 2927 , 2856, 1693, 1454, 1388, 1141, $1090 \mathrm{~cm}^{-1} ;{ }^{1} \mathrm{H}-\mathrm{NMR}$ (500 MHz, $\mathrm{CDCl}_{3}$, two rotamers)

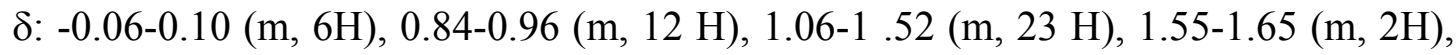
$1.97(\mathrm{~m}, 2 \mathrm{H}$, rotamer 1), 2.12-2.32 (m, 2H, rotamer 2), 2.46-2.60 (m, $1 \mathrm{H}), 2.70-2.82$ (m, $2 \mathrm{H}$, rotamer 1), $3.00(\mathrm{dd}, J=13.6,4.7 \mathrm{~Hz}, 2 \mathrm{H}$, rotamer 2), 3.48-3.71 (m, $1 \mathrm{H})$, 
3.96-4.08 (m, 1H), 4.12-4.20 (m, $2 \mathrm{H}), 4.25$ (dd, $J=16.7,7.1 \mathrm{~Hz}, 2 \mathrm{H}), 7.10-7.30$ (m, $5 \mathrm{H}) ;{ }^{13} \mathrm{C}$-NMR $\left(125 \mathrm{MHz}, \mathrm{CDCl}_{3}\right.$, two rotamers) $\delta: 154.9,140.1,129.9,128.0$, 125.6, 78.9, 71.4, 62.3, 55.7, 38.0, 37.2, 35.9, 31.9, 29.7, 29.5, 29.3, 28.0, 26.5, 25.85, 22.7, 18.1, 14.1, -4.7, -5.0; MS (ESI): $540\left(\mathrm{M}+\mathrm{Na}^{+}, 74\right), 518\left(\mathrm{M}+\mathrm{H}^{+}, 100\right), 404(83)$.

(2S, 3S, 5R)- 2-Benzyl-1-methyl-5-(n-nonyl)-3-pyrrolidinol (Preussin, 5)

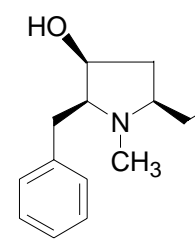

To a solution of 20 (34 mg, $0.084 \mathrm{mmol})$ in dry THF $(0.85 \mathrm{~mL})$ was added $\mathrm{LiAlH}_{4}(32$ $\mathrm{mg}, 0.84 \mathrm{mmol}$ ) in one portion. The resulting reaction mixture was stirred at $60{ }^{\circ} \mathrm{C}$ for $28 \mathrm{~h}$. After being diluted with $2 \mathrm{~mL}$ of $\mathrm{Et}_{2} \mathrm{O}, \mathrm{Na}_{2} \mathrm{SO}_{4} \cdot 10 \mathrm{H}_{2} \mathrm{O}$ was added to quench the reaction. After filtration and removal of the solvent under reduced pressure, the crude was purified by chromatography on silica gel $($ EtOAc / $\mathrm{PE}=1 / 5)$ to give preussin (5) $(24 \mathrm{mg}, 90 \%)$ as a colorless oil. $[\alpha]_{\mathrm{D}}{ }^{20}+21.9\left(c 1.3, \mathrm{CHCl}_{3}\right)\left[\right.$ natural $\mathbf{5},[\alpha]_{\mathrm{D}}^{25}+22.0$ (c 1.0, $\left.\mathrm{CHCl}_{3}\right)$ ]; IR (KBr) v $v_{\max }: 3435,2925,2854,1455,1457,1391,1174 \mathrm{~cm}^{-1}$; ${ }^{1} \mathrm{H}-\mathrm{NMR}\left(500 \mathrm{MHz}, \mathrm{CDCl}_{3}\right) \delta: 0.88(\mathrm{t}, J=6.91 \mathrm{~Hz}, 3 \mathrm{H}), 1.16-1.38(\mathrm{~m}, 15 \mathrm{H})$, 1.38-1.46 (m, $1 \mathrm{H}), 1.72(\mathrm{~m}, 1 \mathrm{H}), 2.11(\mathrm{~m}, 1 \mathrm{H}), 2.18(\mathrm{~m}, 1 \mathrm{H}), 2.26(\mathrm{~m}, 1 \mathrm{H}), 2.33(\mathrm{~s}$, $3 \mathrm{H}), 2.80-2.92(\mathrm{~m}, 2 \mathrm{H}), 3.8(\mathrm{~m}, 1 \mathrm{H}), 7.15-7.35(\mathrm{~m}, 5 \mathrm{H}) ;{ }^{13} \mathrm{C}-\mathrm{NMR}(125 \mathrm{MHz}$, $\left.\mathrm{CDCl}_{3}\right) \delta: 139.5,129.4,128.4,126.1,73.5,70.5,65.7,39.3,38.7,35.1,33.7,31.9$, 29.9, 29.6, 29.6, 29.3, 26.3, 22.7, 14.1; MS (m/z): $318\left(\mathrm{M}+\mathrm{H}^{+}, 100\right)$; HRMS calcd for $\left[\mathrm{C}_{21} \mathrm{H}_{35} \mathrm{NO}+\mathrm{H}\right]^{+}: 318.2791$; found: 318.2786 .

(3S, 4S)-4-tert-Butyloxycarbonylamino-3-hydroxy-5-phenylpentanoic acid ethyl ester (21)

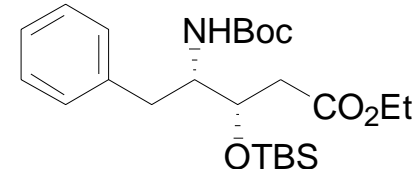

To a solution of $19(10 \mathrm{mg}, 0.025 \mathrm{mmol})$ in dry THF $(0.1 \mathrm{~mL})$ was added a catalytic amount of potassium cyanide and EtOH $(0.1 \mathrm{~mL})$. The mixture was stirred at $\mathrm{rt}$ for 16 $\mathrm{h}$. The solvent was removed under reduced pressure and the crude was purified by column chromatography on silica gel (EtOAc / PE= 1/12) to give $21(6 \mathrm{mg}, 91 \%)$ as a colorless oil. $[\alpha]_{\mathrm{D}}{ }^{20}-23.4(c 0.7, \mathrm{MeOH})$; IR $(\mathrm{KBr}) v_{\max }: 2956,2930,2857,1737$, 1703, 1366, 1253, $1174 \mathrm{~cm}^{-1}$; ${ }^{1} \mathrm{H}-\mathrm{NMR}\left(500 \mathrm{MHz}, \mathrm{CDCl}_{3}\right.$, two rotamers, $\mathrm{M}$ and $\left.\mathrm{m}\right) \delta$ : $0.02(\mathrm{~s}, 3 \mathrm{H}), 0.07(\mathrm{~s}, 3 \mathrm{H}), 0.92(\mathrm{~s}, 9 \mathrm{H}), 1.23(\mathrm{t}, J=6.9 \mathrm{~Hz}, 3 \mathrm{H}), 1.35$ (s, $9 \mathrm{H}), 2.47$ (dd, $J=15.9,6.0 \mathrm{~Hz} 1 \mathrm{H}, \mathrm{M}), 2.51-2.59(\mathrm{dd}, J=15.9,6.0 \mathrm{~Hz} 1 \mathrm{H}, \mathrm{m}), 2.68-2.77$ (m, $2 \mathrm{H}$, m), 2.78-2.90 (m, $2 \mathrm{H}, \mathrm{M}), 3.80-3.87(\mathrm{~m}, 1 \mathrm{H}, \mathrm{m}), 3.92-3.99(\mathrm{~m}, 1 \mathrm{H}, \mathrm{M}), 4.12(\mathrm{q}, J=$ $6.9 \mathrm{~Hz}, 2 \mathrm{H}, \mathrm{M}), 4.18-4.28$ (m, 2H, m), 4.55 (brs, $1 \mathrm{H}, \mathrm{m}), 4.62-4.68(\mathrm{~m}, 1 \mathrm{H}, \mathrm{M})$, 7.15-7.35 (m, $5 \mathrm{H}) ;{ }^{13} \mathrm{C}-\mathrm{NMR}\left(125 \mathrm{MHz}, \mathrm{CDCl}_{3}\right.$, two rotamers) $\delta: 171.2,155.5$, 138.2 , 129.1, 128.3, 126.2, 79.1, 69.8, 60.5, 55.4, 39.9, 38.5, 28.3, 25.9, 18.1, 14.1, 
